\title{
Configuration Study of Structurally Integrated Thermal Protection Systems for a Sub-Orbital Platform
}

\author{
José Guido Damilano¹, Humberto Araújo Machado1,2, Domingos Sávio Aguiar, Fabio Eduardo de \\ Almeida ${ }^{1}$, José Antônio Azevedo Duarte ${ }^{1}$, João Luiz Filgueiras de Azevedo ${ }^{1}$
}

\begin{abstract}
The atmospheric hypersonic flight of sub-orbital and space vehicles generates aerodynamic heating and high wall heat fluxes, inducing high temperatures on the vehicle's structures and affecting their mechanical behavior, besides degrading the operation of board equipment. Furthermore, since payload preservation is always mandatory, the use of efficient Thermal Protection Systems (TPS) is a key-requirement for any spacecraft design. As an outcome, designing the TPS is a critical aspect of any rocket development program, since an undersized system may result in catastrophic failure, and an oversized one implies increased mass and cost. Sub-orbital platforms are a low-cost alternative for microgravity research. A sub-orbital platform (SARA) is being developed by Instituto de Aeronáutica e Espaço (IAE) for such an application, and its current design uses a conventional layer of cork as TPS to protect its lateral surface, with the trade-off of large mass. Alternatively, a Thermally Integrated Structural Sandwich Core [TISSC), which consists of a structural sandwich panel in a three-layer plate with two face sheets and the core, presents advantages such as lightweight, low maintenance, insulation as well as load bearing capabilities, and low life-cycle cost. In this work, a TISSC is proposed to replace SARA's current TPS. The main contribution of the presented methodology is to couple the aerodynamic heating, heat transfer in porous insulation and thermo-structural analyses of the proposed configuration in order to evaluate the TISSC TPS performance. The results are compared with those obtained for the current SARA TPS design, showing improvements in thermal insulation and structural strength, as well as a remarkable mass reduction
\end{abstract}

KEYWORDS: Thermal protection system, Aerodynamic heating, Thermo-structural analysis, Fibrous insulation, Computational simulation.

\section{INTRODUCTION}

Space vehicles reach very high velocities during atmospheric flight, inducing aerodynamic heating (Anderson, 1989) due to a supersonic shock wave in the vicinity of the vehicle's wall and due to friction against the air molecules. As a result, heat exchange by convection and radiation between the heated air and the vehicle's wall is part of the problems faced in atmospheric flight. In case of recoverable vehicles, aerodynamic heating occurs throughout the launching phase as well as in the re-entry phase. In the latter, since the velocities are higher, the heating intensity is such that temperatures over $1,000^{\circ} \mathrm{C}$ are obtained at the spacecraft's stagnation point (Machado and Pessoa Filho, 2007; Machado, 2009). The high temperatures negatively affect both the structure's bearing load capacity and the payload integrity. Thus, including a thermal protection system (TPS) on the spacecraft is mandatory. A careful design of an efficient thermal protection system is paramount to the spacecraft's mission completion, since an undersized system may result in catastrophic failure, and an oversized one implies increased mass and cost (Moraes Jr., 1998).

A common way of thermally protecting a spacecraft system is through the inclusion of an ablating surface that will experience phase changes during flight, absorbing the heat generated in the process (Rogan and Hurwicz, 1973). Among the commonly used ablative materials, there are several types of composites (fibers and resins). When the surface is ablated, the resin experiences a series of chemicals reactions releasing gaseous byproducts (pyrolysis), leaving a layer of char, right after the pyrolysis front, through which

\footnotetext{
1. Departamento de Ciência e Tecnologia Aeroespacial - Instituto de Aeronáutica e Espaço - Divisão de Sistemas Espaciais - São José dos Campos/SP - Brazil. 2. Universidade do Estado do Rio de Janeiro - Faculdade de Tecnologia de Resende - Resende/RJ - Brazil.

Author for correspondence: Humberto Araújo Machado | Instituto de Aeronáutica e Espaço | Praça Eduardo Gomes, 50 - Vila das Acácias | CEP: $12.228-901$ São José dos Campos/SP - Brazil | Email: humbaman@uol.com.br Received: 10/06/2014 | Accepted: 02/28/2015
} 
these thermo-chemical processes result in gases flow (da Costa et al., 1996). In the regions where heat loads and air temperature are lower, the surface warming is less critical, and a thermal insulation layer can be employed. Such thermal protection should be able to prevent or reduce the two main heat transfer modes involved, convection and radiation. Currently-used TPS includes multilayer coatings composed of insulating and reflexive materials (Pessoa Filho and Genaro, 2006). Also, fibrous ceramic tiles over flexible material bases are used, as in the NASA space shuttles, for example.

For the next generation of recoverable space vehicles, the use of metallic thermal protection systems has been considered. It comprises a high-temperature resistant metallic alloy integrated into the spacecraft structure, filled with a light weight fibrous material. The specific configuration, namely Thermally Integrated Structural Sandwich Core (TISSC), would insulate the vehicle from aerodynamic heating, and it would be also capable of carrying primary vehicle loads. TISSC consists of a structural sandwich panel in a three-layer plate with two face sheets and the core. Two thin, stiff and strong faces are separated by a thick and light weight core (Poteet et al., 2004). It shows promising perspectives for application, since the light weight fibrous material guarantees excellent thermal insulation and the metallic frame increases structural strength (Fig. 1). Its main advantages are: highly efficient thermal insulation; very low maintenance; structural robustness; thermal and structural integration with the spacecraft main body. Nevertheless, for the correct design and sizing of such systems, some technical difficulties must be addressed: induced thermal stress due to the integration of different materials; balance between thermal and structural performance; definition of the most appropriate material; construction and integration into the vehicle structure. Therefore, the starting point for the optimization of a TPS structurally integrated is the knowledge about the thermo-structural behavior of both the structure and the insulating fibrous material working in a synergistic manner.

Several formulations to study heat transfer on insulating fibrous materials have been proposed (Raed and Gross, 2007), and several studies have been carried out towards modeling and optimizing the structural design of active thermal protection systems (Tamma and Thronton, 1987; Rakow and Wass, 2005). Nevertheless, combining insulating fibrous materials with metallic structural components of a TPS still requires investigations that could precisely predict the thermo-structural behavior of the system.

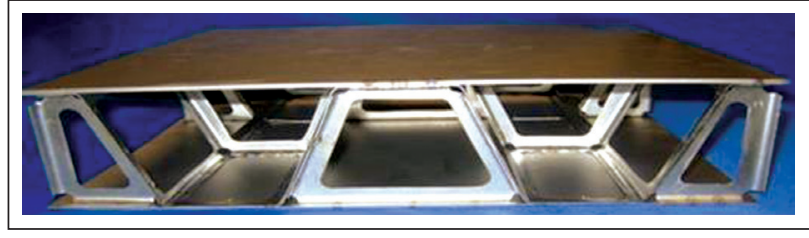

Figure 1. TPS structurally integrated

This paper aims at developing a methodology for modeling the thermo-structural behavior of structural thermal protection systems and the application of such systems. Also, aims at applying an integrated methodology for modeling the thermostructural behavior of TISSC, where the aerodynamic heating, heat transfer in porous insulation and thermo-structural analyses are coupled in sequence, which allows to obtain the temperature and the stress distribution for the whole trajectory of a vehicle, not only for particular instants or conditions like in the previously mentioned studies.

The proposed methodology is applied on sizing a structural thermal protection system for the SARA sub-orbital platform, a recovery sub-orbital vehicle that has been developed for microgravity experiments, in order to obtain the resulting performance and possible TPS mass reduction when compared with the conventional TPS originally designed.

\section{MATHEMATICAL MODEL AERODYNAMIC HEATING}

To predict the heat transfer on SARA, it is necessary to know pressure, temperature and velocity fields around the vehicle. This can be accomplished by numerically solving the $\mathrm{N}-\mathrm{S}$ equations. However, such a procedure is expensive and time-consuming. In the present work, a simpler, but reliable, engineering approach is also used. The following simplifying assumptions are made:

- Zero angle of attack.

- SARA rotation around its longitudinal axis is neglected.

- Atmospheric air is considered to behave as a calorically and thermally perfect gas (no chemical reactions).

The free stream conditions ahead of the nose cap are those given by $v_{\infty}, T_{\infty}, p_{\infty}$, corresponding, respectively, to velocity, temperature and pressure. By knowing $v_{\infty}$ and altitude, as function of time, together with an atmospheric model (National Oceanic and Atmospheric Administration, 1976), it is possible to evaluate the free stream properties, such as $p_{\infty}, T_{\infty}$, and $c_{\infty}$, which represent free stream pressure, temperature and speed of sound, respectively. 
For supersonic flow $\left(M_{\infty}>1\right)$, a detached shock wave appears ahead of the nose. By using the normal shock relationships, it is possible to calculate $v_{1}, T_{1}$ and $p_{1}$ after the shock.

The heat flux over the external surface was calculated through the Zoby's method (Zoby et al., 1981), namely:

$$
q=h\left(T_{a w}-T_{w}\right)
$$

where:

$h$ : convective heat transfer coefficient; $q$ : heat flux; $T_{w}$ : wall temperature.

The adiabatic wall temperature, $T_{a w}$, also called recovery temperature, $T_{r}$, is given by:

$$
T_{a w}=T_{e}+F_{R} \frac{v_{e}^{2}}{2 c_{p}}
$$

where:

$c_{p}$ : specific heat at constant pressure; $T_{e}$ : temperature; $v_{e}$ : velocity; $e$ (subscript): refers to conditions at the boundary layer edge; $F_{R}$ : recovery factor, equal to $\sqrt{P r_{w}}$, for laminar flow and $\sqrt[3]{P r_{w}}$ for turbulent flow; $P r_{w}$ : Prandtl number evaluated at wall temperature, $\operatorname{Pr}_{w}=0.71$.

The convective heat transfer coefficient comes from the Reynolds analogy, namely:

$$
h=0.5 \rho_{e} c_{p} v_{e} \operatorname{Pr}_{w}^{-a} C_{F}
$$

where:

$a$ is equal to 0.6 for laminar flow and 0.4 for turbulent flow; $\rho$ : specific mass.

To take into account compressibility effects, a modified friction factor is obtained:

$$
C_{F}=K_{1}\left(R e_{\theta}\right)^{K_{2}}\left(\frac{\rho_{e}^{*}}{\rho_{e}}\right)\left(\frac{\mu_{e}^{*}}{\mu_{e}}\right)^{K_{3}}
$$

where:

$K$ : thermal conductivity; $\mu$ : viscosity; ${ }^{\star}$ (superscript): refers to properties evaluated at Eckert's reference temperature $\left(T_{e}^{*}\right) ; R e_{\theta}$ : Reynolds number, based on the boundary layer thickness $(\theta)$ :

$$
R e_{\theta}=\frac{\rho_{e} v_{e} \theta}{\mu_{e}}
$$

Viscosity, $\mu$, is evaluated according to Sutherland's equation, as function of temperature (Anderson, 1989). In Eq. 4, $K_{1}=0.44, K_{2}=-1$ and $K_{3}=1$, for laminar flow. For turbulent flow, $K_{2}=K_{3}=-m$, and

$$
\begin{aligned}
& K_{1}=2\left(\frac{1}{C_{5}}\right)^{\frac{2 N}{N+1}}\left[\frac{N}{(N+1)(N+2)}\right]^{m} \\
& m=\frac{2}{N+1} \\
& C_{5}=2.2433+0.93 \mathrm{~N} \\
& N=12.76-6.5 \log _{10}\left(R e_{\theta}\right)+1.21\left[\log _{10}\left(R e_{\theta}\right)\right]^{2}
\end{aligned}
$$

For laminar flow, the boundary layer thickness is given by:

$$
\theta_{L}=\frac{0.664\left(\int_{0}^{y} \rho_{e}^{*} \mu_{e}^{*} v_{e} R^{2} d y^{\prime}\right)^{\frac{1}{2}}}{\rho_{e} v_{e} R}
$$

where:

$y$ (tangential coordinate) measured along the body's surface and $y=0$ corresponds to the stagnation point; $R$ : geometric parameter.

In this study, the numerical integration of Eq. 7 was obtained according to the trapezoidal method. As $R \rightarrow 0$, Eq. 7 becomes undetermined. By taking the limit of Eq. 7 as $R \rightarrow 0$, the following expression is obtained:

$$
\theta_{L}=\frac{0.332\left(\rho_{e}^{*} \mu_{e}^{*}\right)^{\frac{1}{2}}}{\sqrt{\frac{1}{R_{N}}\left[\frac{2\left(p_{s}-p_{\infty}\right)}{p_{s}}\right]^{\frac{1}{2}}}}
$$

In this study, Eq. 8 is applied for $y<0.1 R_{N}$, where $R_{N}$ is the curvature radius at the stagnation point.

The boundary layer thickness for turbulent flow is obtained by solving the following first-order differential equation:

$$
\frac{D\left(\rho_{e} v_{e} R \theta_{T}\right)}{D y}=0.5 C_{F} \rho_{e} v_{e} R
$$

After obtaining the boundary layer momentum thickness, $\theta, \operatorname{Re}_{\theta}, C_{F}$ and $h$ can be evaluated by using Eqs. 5, 4 and 3, respectively. Along the transition region between laminar 
and turbulent flow, the following relationship is used (Zoby et al., 1981):

$$
q_{T r}=q_{L}+F(y)\left(q_{T}-q_{L}\right)
$$

where the subscripts $\operatorname{Tr}, L$ and $T$ represent, respectively, transitional, laminar and turbulent flow. The transitional factor, $F(y)$, is given by:

$$
F(y)=1-\exp \left\{-0.412\left[\frac{4.74\left(y-y_{L}\right)}{\left(y_{T}-y_{L}\right)}\right]\right\}
$$

Transition is supposed to occur for $163<R e_{\theta}<275$.

Property evaluation at the boundary layer edge is performed assuming isentropic flow between the stagnation region and the location $i$, where properties are needed, namely:

$$
\begin{aligned}
& \rho_{e, i}=\rho_{s}\left(\frac{p_{e, i}}{p_{s}}\right)^{\frac{1}{\gamma}} ; h_{e, i}=h_{s}\left(\frac{p_{e, i}}{p_{s}}\right)^{\frac{\gamma-1}{\gamma}} ; \\
& v_{e, i}=\sqrt{2\left(h_{s}-h_{e, i}\right)} ; T_{e, i}=\left(\frac{h_{e, i}}{c_{p}}\right)
\end{aligned}
$$

where:

$i$ : discrete position in which the parameter is calculated along the surface. At the stagnation point, $i=1$.

The local pressure, $p_{e, i}$, is obtained from the modified Newton method and $\gamma=1.4$. The results of both methods are then compared. The subscript $s$ appearing in Eq. 12 refers to the stagnation condition. Eckert reference temperature is obtained from:

$$
\frac{T_{e, i}^{*}}{T_{e, i}}=1+0.032 M^{2}{ }_{e, i}+0.58\left(\frac{T_{w}}{T_{e, i}}-1\right)
$$

The solution procedure can be summarized as follows:

- From a given trajectory, the US Standard Atmosphere (National Oceanic and Atmospheric Administration, 1976) is used to obtain the free stream properties, including the stagnation ones.

- Normal shock relationships are used to obtain the fluid flow properties behind the shock.

- By using the modified Newton method, pressure distribution is obtained along the payload.

- Equation 12 provides the local properties at the boundary layer edge.
- If $y<0.1 R_{N}$, Eq. 8 provides the laminar boundary layer thickness, leading to the estimation of $R e_{\theta}, C_{F}$ and $h$, provided by Eqs. 5, 4 and 3, respectively.

- If $y>0.1 R_{N}$ and $R e_{\theta}<163$, Eq. 7 is numerically integrated up to the location where the momentum thickness is to be estimated. Such an integration is performed by using the trapezoidal method.

- If $y>0.1 R_{N}$ and $R e_{\theta}>275$, Eq. 9 is numerically integrated by the trapezoidal rule leading to the turbulent boundary layer thickness.

- If $y>0.1 R_{N}$ and $163<R e_{\theta}<275$, Eqs. 10 and 11 are used to estimate $h$.

It should be pointed out that such a procedure is performed along the payload's surface (following the $y$-coordinate), for different trajectory times. Therefore, $h=h(y, t)$.

\section{HEAT TRANSFER IN FIBROUS INSULATION}

Once the film coefficient and the external air temperature along the trajectory are known, the temperatures on the wall are calculated through the solution of the energy equation applied to the process of heat conduction on the TISSC system. Since the wall is made with aluminum, which has a high thermal diffusivity, it can be treated as a one-dimensional problem along the wall thickness (Machado, 2006). Here, the thermo-physical properties of the system's several layers must be known. For the insulating fibrous material inside the structural lattice, the heat conduction process involves: conduction through the fibers; conduction through the gases retained within the fibers' voids; radiant heat exchange in participating media (fiber and gases); and, possibly, some natural convection.

Environmental pressures varying from $1.33 \times 10^{-5}$ to $101.32 \mathrm{kPa}$ and temperatures varying from 300 to $1,300 \mathrm{~K}$ act on the insulating fibrous material. Most of the heat transfer models used for insulating fibrous materials have been validated through experimental results, obtained at limited intervals of pressure and temperature (Daryabeigi, 2003). The mathematical model herein applied (Daryabeigi, 2000, 2002, 2003; Daryabeigi et al., 2006, 2007) considers heat transfer between two plates by combining the radiation and conduction modes. The insulating fibrous material between the plates is considered a articipating media. The main equation resulting from this approach is the one-dimensional energy conservation equation with an added source term for the radiant heat exchange.

$$
\rho c \frac{\partial T}{\partial t}=\frac{\partial}{\partial y}\left(k_{c} \frac{\partial T}{\partial y}\right)=\frac{\partial q_{r}^{\prime \prime}}{\partial y}
$$


where:

$\rho c=\rho c p ; k_{c}$ : thermal conductivity of the solid; $r$ (subscript): related to radiation.

The equivalent thermal conductivity model, for the porous media, was validated through Daryabeigi's experimental results (Daryabeigi, 2003) and a modified version of the model described in this work, proposed by Machado (2014), which used Saffil $®$ (alumina fiber) as the porous media.

For an optically thick medium, the diffusion approximation can be used, resulting in the radiant heat flux of:

$q_{r}^{\prime \prime}=-k_{r} \frac{\partial T}{\partial y}$

Using this approximation, the energy equation reduces to:

$\rho c \frac{\partial T}{\partial t}=\frac{\partial}{\partial y}\left(k_{e f f} \frac{\partial T}{\partial y}\right)$

where:

$k_{\text {eff }}$ : apparent or effective thermal conductivity.

$k_{e f f}$ is obtained by superposition of the thermal conductivities due to solid conduction, gas conduction, and radiation:

$k_{e f f}=k_{r}+k_{s}+k_{g}$

where:

$k_{r}$ : radiant thermal conductivity for fibrous insulations; $k_{s}$ : solid conduction term; $k_{g}$ : thermal conductivity of the gas.

The radiant thermal conductivity for fibrous insulations, $k_{r}$, is provided by:

$$
k_{r}=\frac{16 \sigma n^{* 2} T^{3}}{3 \rho e}
$$

where:

$\sigma$ : Stefan-Boltzman constant $\left(5.670 \times 10^{-8} \mathrm{~W} / \mathrm{m}^{2} \mathrm{~K}^{4}\right)$; $e$ : specific extinction coefficient, estimated from the experimental data using a genetic algorithm-based parameter estimation technique; $n^{*}$ : effective index of refraction.

A curve fit of the effective index of refraction with fiber volume fraction was used. The optical thickness is given by $\tau=\rho e L$, where $L$ is the thickness of the fibrous insulation. The insulation can be considered optically thick only if $\tau>>1$, which is valid for insulation samples used in this study with optical thickness $>20$.
The solid conduction term, $k_{s}$, is extracted from an empirical model based on density and thermal conductivity, $k$, of bulk material:

$$
k_{s}(T)=F_{s} f_{v}^{b} k_{s}^{*}(T), \quad 1 \leq b \leq 3
$$

where:

$F_{s}$ : factor relating microscale geometric effects of fiber matrix and bulk dimensions. It is obtained from steady state measurements in vacuum and at cryogenic temperatures (test condition with negligible gas conduction and reduced radiation); $B$ : constant not known a priori; $f_{v}$ : volume fraction.

The heat conduction is considered to occur in the porous media, as a combination of solid and gas conduction processes. The solid conduction occurs through the fibrous, and its thermal conductivity dependence on temperature is considered to be well-known.

According to statistic thermodynamics, gas thermal conductivity is supposed to depend only on the temperature and to be independent on the pressure. However, in a porous media, it was verified that pressure plays an important role below a certain level.

The current model uses a classical approach to estimate the gas thermal conductivity inside the fibrous, based on the Knudsen hypothesis. The standard temperature function used to estimate the gas thermal conductivity, $k_{g 0}(T)$, is corrected through the following equation (Raed and Gross, 2007):

$$
k_{g}=\frac{k_{g 0}(T)}{\Phi+\Psi \frac{\beta}{P r} K n}
$$

where:

$\Phi=1, \psi=0$ for $K n<0.01$ (continuum regime); $\Phi=1$, $\psi=1$ for $0.01<K n<10$ (transition regime); $\Phi=0, \psi=1$ for $K n>10$ (free-molecular regime); and $\beta$ :

$$
\beta=\left(\frac{2-\alpha}{\alpha}\right)\left(\frac{2 \gamma}{2 \gamma+1}\right)
$$

where:

$\alpha$ : thermal accommodation coefficient; $\gamma$ : gas specific heat ratio; $K n$ : Knudsen number, being:

$$
K n=\frac{\lambda}{L_{C}}
$$


where:

$\lambda$ : molecular mean free path of the gas; $L_{C}$ : characteristic length of the fibrous insulation.

This model does not include the possibility of heat convection within the fibrous insulation. However, in microscale, it is remarkable that the two phases of the porous media, fibers and gas, have different optical properties. The gas is considered to be transparent, and the solid fibers approach to the behavior of a black body. Indeed, any radiative heat transfer to the media will cause different heating in each phase, and the temperature reached in the fibers tends to be higher than in the gas. This difference will depend on several parameters, but no matter how small, it can cause a local heat exchange by natural convection between the fiber and the gas. Since it occurs in microscale, as soon as the heated gas gets far from the fiber surface, it mixes with the bulk gas around, reaching thermal equilibrium and ceasing the natural convection. The distance traveled by the gas until stopping may correspond to a few fiber diameters. It means that the process occurs continuously in the domain and it is as homogeneous as the fiber distribution, approaching a diffusion process. This hypothesis explains the fact that the values found for this parameter in the experimental tests are higher than the theoretical ones in the majority of the works; this difference appears and rises with the pressure increasing. When this effect is accounted, the effective thermal conductivity is better represented by:

$k_{e f f}=k_{r}+k_{s}+(1-f)\left(k_{g}+h D\right)$

where:

$D$ : fiber diameter.

A detailed model for obtaining $h$ and the comparison between the original and modified models, showing the improvements of the new model when compared with experimental results, were presented by Machado (2014).

According to this model, the variation of $k_{e f f}$ as function of pressure and temperature difference between the plates (with a lower temperature of $60^{\circ} \mathrm{C}$, which is the limit for the internal surface) is showed in Fig. 2.

Both variations can be fitted by the following function:

$$
\begin{aligned}
& k_{e f f}(\Delta T, P)=F(P) \mathrm{G}(\Delta T) \\
& G(\Delta T)=b e^{a \Delta T}
\end{aligned}
$$

where:

$a=0.001884858198$ and $b=0.03446775065$.

$F(P)=b P^{a}$

where:

$a=0.005102720025$ and $b=0.03910708429$. This function allows obtaining an overall $\mathrm{rms}$ error of $1.33 \%$ when compared to the model results.

In order to extract the other physical properties of the porous media, the following equations were employed:

$$
\begin{aligned}
& c P_{\text {air }}=1027.582-0.2216 T+4.561 \times 10^{-4} T^{2}+ \\
& 2.455 \times 10^{-7} T^{3}-16.643 \times 10^{-10} T^{4}+3.616 \times 10^{-13} T^{5}- \\
& 6.349 \times 10^{-17} T^{6}
\end{aligned}
$$$$
K_{\text {air }}=-1.8763 \times 10^{-3}+1.218 \times 10^{-4} \mathrm{~T}-1.328 \times 10^{-2} \mathrm{~T}^{2}
$$$$
+1.479 \times 10^{-10} T^{3}-18.58 \times 10^{-14} T^{4}+1.705 \times 10^{-17} T^{5}
$$$$
+9.527 \times 10^{-22} T^{6}
$$

$$
K_{s}=\left(1.63 \times 10^{-5}-2.114 \times 10^{-8} T+1.131 \times 10^{-11} T^{2}-\right.
$$
$\left.2.094 \times 10^{-15} T^{3}\right) \times \rho$

where:

$\rho$ is the density of the porous material. In this work, $\rho$ was considered to be $24.2 \mathrm{~kg} / \mathrm{m}^{3}$.

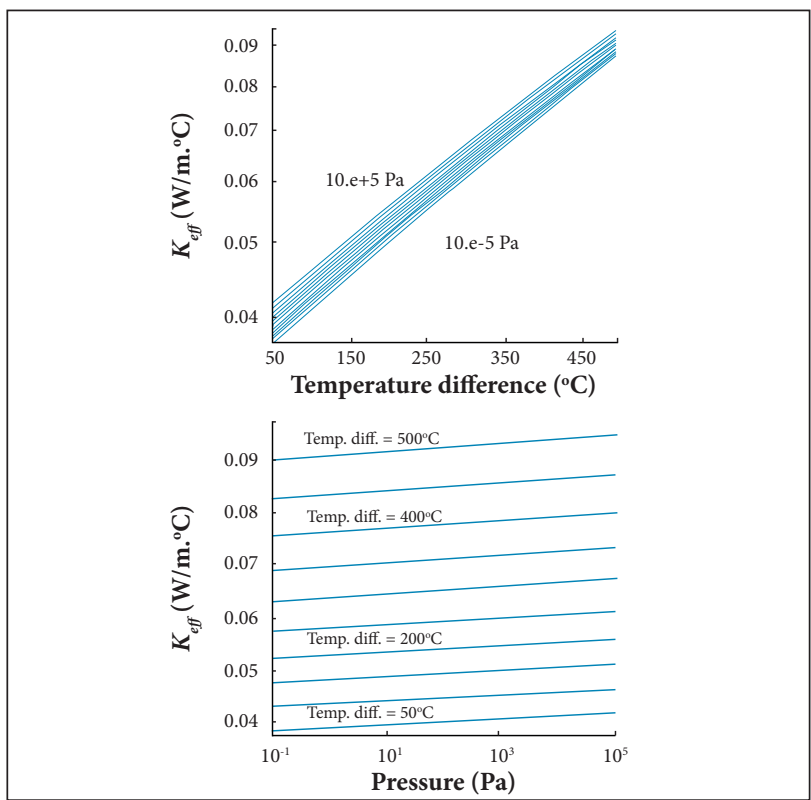

Figure 2. Results for Keq as function of pressure and temperature difference. 


\section{STRUCTURAL ANALYSIS}

The structural model studied is presented in Fig. 3. The void regions are filled with the insulating fibrous material. A 3-D finite element model, with tetrahedron elements, is used to evaluate temperature distribution, displacements, strains and stress on the structure by using the finite element software ABAQUS®.

The model's upper plate represents the vehicle's wall external surface receiving the aerodynamic heating. On the other hand, the lower plate represents the vehicle's internal region which must maintain the temperatures defined by the design $\left(60^{\circ} \mathrm{C}\right)$. The heat flux, temperatures and thermal conductivities calculated with the aerodynamic heating and heat conduction models, respectively, are then applied on the structural model resulting in displacements, strains, stresses, and temperature distributions at each point within the structure.

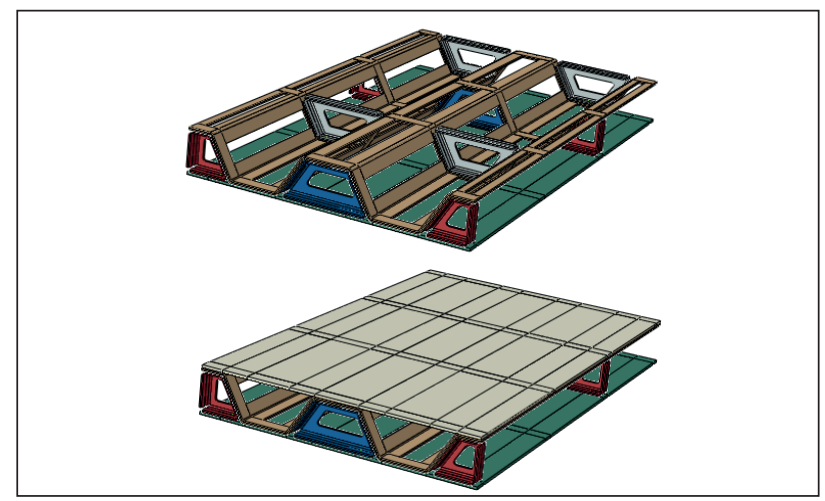

Figure 3. TISSC structural model without (top) and with (bottom) the upper plate.

\section{RESULTS}

The sub-orbital platform SARA was initially selected for the studies using the methodology presented in this work. Sub-orbital platforms are low cost alternatives for microgravity research. SARA's total mass will be of the order of $250 \mathrm{~kg}$ with a payload mass of about $25 \mathrm{~kg}$, and it will provide 6 minutes of micro-gravity environment. SARA may reach speeds of $9,300 \mathrm{~km} / \mathrm{h}$ in atmospheric flight. A future orbital version of SARA may reach an orbit of $300 \mathrm{~km}$ and an orbital period of 10 days (Moraes Jr., 1998).

Figure 4a shows SARA's lateral thermal protection system in its original configuration. A structural aluminum wall $4 \mathrm{~mm}$ thick is covered with a $6 \mathrm{~mm}$ thick cork layer applied all over the vehicle's conical region. A new configuration, similar to the one shown in Fig. 1, is proposed. The system is a sandwich structure composed of two $1 \mathrm{~mm}$ thick aluminum walls filled with an $18 \mathrm{~mm}$ layer of Saffil $尺$, shown in Fig. 4b. The total thickness variation is compatible with the geometry of the spherical cap at the top.

The aerodynamic parameter ( $h$ and $T_{R}$ ) calculations presented by Machado (2012) showed results with slight variations along the conical region. Thus, average values at the conical region for the convection heat transfer coefficient $h(\mathrm{t})$ and the recovering temperature $T_{r}(\mathrm{t})$, during the ascending trajectory, were used in this work. Figure 5 shows both curves.

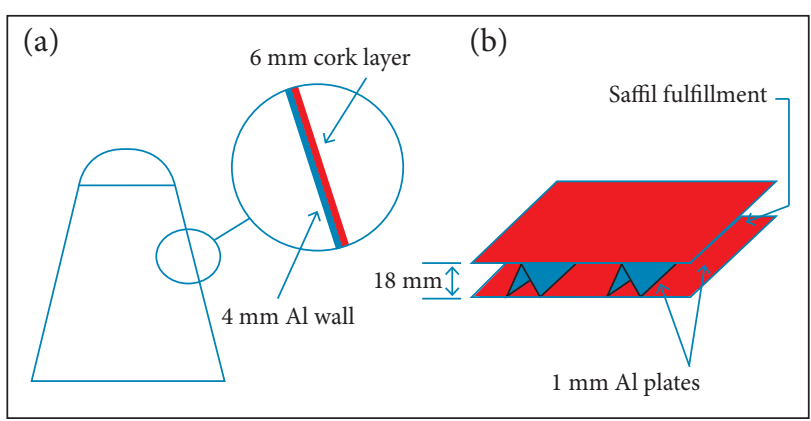

Figure 4. SARA's thermal protection systems. (a) Original configuration; (b) proposed configuration.

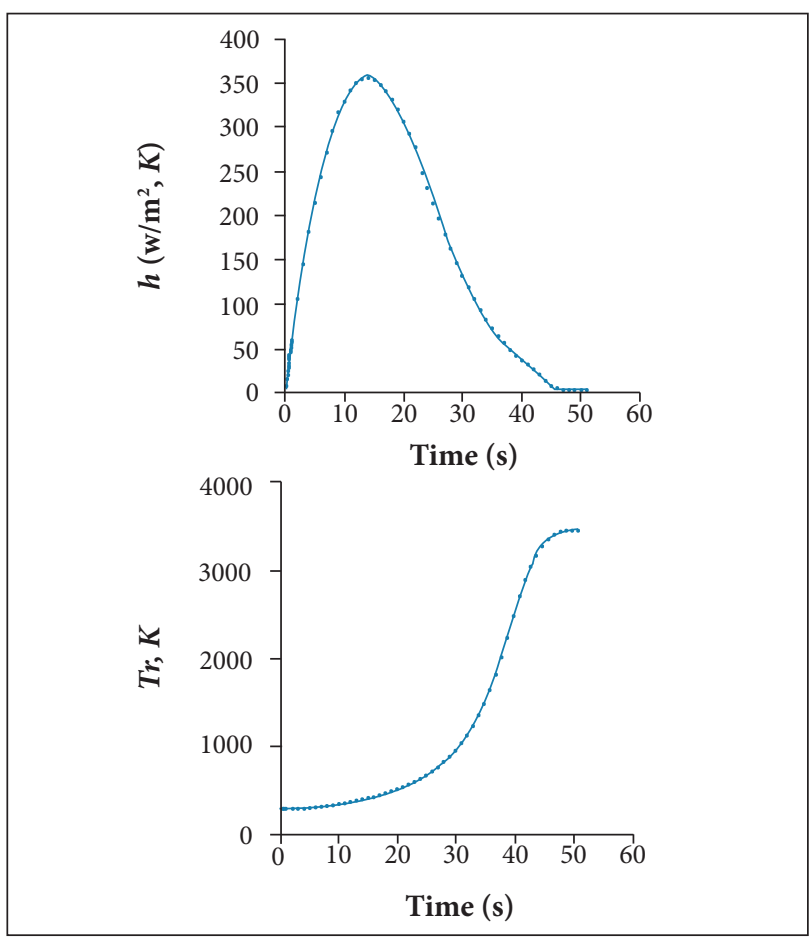

Figure 5. Average values of $h(t)$ and $\operatorname{Tr}(t)$ at SARA's conical region. 
A finite element model using 77,604 tetrahedron elements C3D4T (ABAQUS®) and approximately 15 thousand nodal points was employed in the analyses. Figure 6 shows a section of the mesh, where the red dots indicate nodes with constrained translations in the $x, y$, and $z$ directions. A constant load of 5 atmospheres, corresponding to the maximum dynamic pressure, was applied to the structure. As initial condition, the entire structure is subjected to the temperature of $300 \mathrm{~K}$. The boundary conditions for temperature were Eq. 1 for external surface and adiabatic wall for internal surface. Material properties are presented in Table 1.

Figure 7 brings temperature results for the upper and lower plates. The inner structures connecting the plates work as a heat conductor, thus decreasing the upper external plate temperature and increasing the temperature on the internal lower plate at contact regions. None of the carried out analyses took into account property variations with temperature, as strength and stiffness. Only the values of the thermal conductivity property of the filling fibrous Saffil@ were considered as varying with temperature.

Figures 8, 9 and 10 show temperature distribution over the thermal protection system. It is clear that the internal structural members act as heat conducting devices, transferring the heat from the upper plate to the lower one, inducing a considerable temperature variation on them. Such an effect negatively impacts the insulation capacity

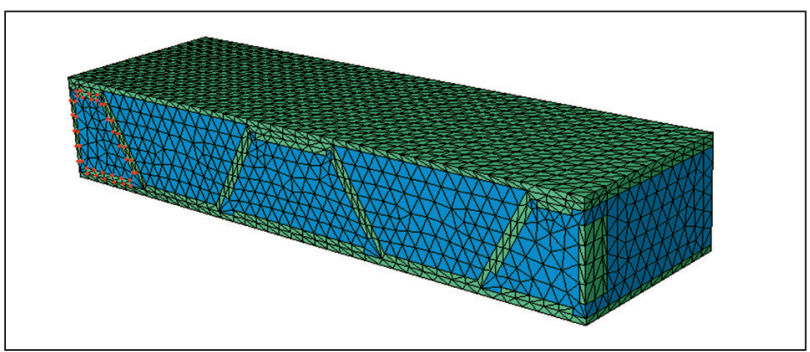

Figure 6. The finite element mesh — front view.

Table 1. Material properties.

\begin{tabular}{|c|c|c|}
\hline \multirow{2}{*}{ Property } & \multicolumn{2}{|c|}{ Material } \\
\cline { 2 - 3 } & Aluminum & Saffil ${ }^{*}$ \\
\hline Young's modulus - E $(\mathrm{GPa})$ & 70 & $3.0 \times 10^{-6}$ \\
\hline Mass density $\rho\left(\mathrm{kg} / \mathrm{m}^{3}\right)$ & 2,700 & 24.2 \\
\hline Specific heat $\left(\mathrm{J} / \mathrm{kg}^{\circ} \mathrm{C}\right)$ & 960 & 1,005 \\
\hline Poisson's ratio $(v)$ & 0.3 & 0.4 \\
\hline Thermal expansion coefficient & $2.0 \times 10^{-5}$ & $8.0 \times 10^{-6}$ \\
\hline Thermal conductivity $\left(\mathrm{W} / \mathrm{m}^{\circ} \mathrm{C}\right)$ & 177 & $*$ \\
\hline *Dependent on temperature and pressure. & & \\
\hline
\end{tabular}

of the system. Nevertheless, this problem may be solved by using different materials for each component, with the inner structure working as heating insulators. A possibility will be the use of composites for those members.

The thermal protection system currently proposed to be used on the SARA platform (Fig. 4a) and the one proposed in this study (Figs. $4 \mathrm{~b}$ and 6 ) had their load carrying capabilities compared. For both designs with the same lateral dimensions as those on Fig. 6, the present model shows a mass reduction of $7.6 \%$. Figures 11 and 12 show displacements and stress results, respectively, when the structure is subjected to an external pressure of 5 atmospheres (maximum dynamic pressure during the flight). The present model shows a reduction in displacements of $30 \%$ and an increase in stress of $62.3 \%$. However, it is important to note that linear tetrahedron elements present very poor stress results, and thus the actual values may be quite different than the ones obtained. Nevertheless, the displacement results are precise enough, and the aim of the present work is not to obtain accurate results for stresses, but to study only both models' load carrying capabilities. The results clearly indicate the proposed design's high stiffness. Obviously, there is still margin to reduce the model stiffness, further reducing the total mass of the whole structural system.

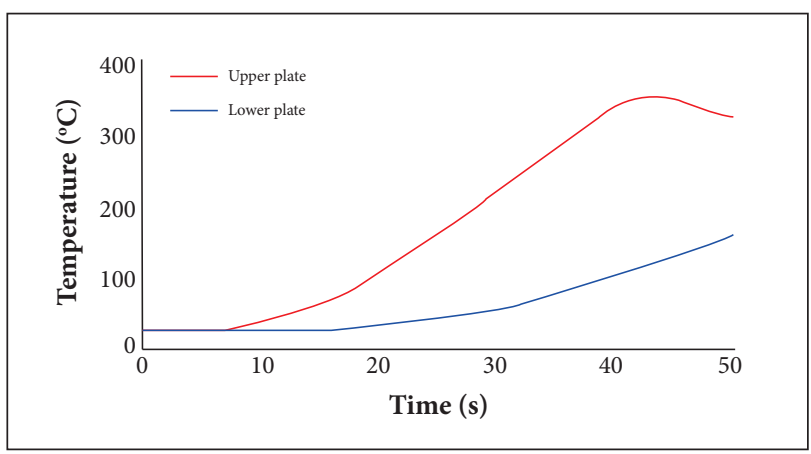

Figure 7. Resulting temperatures on the upper and lower plates.

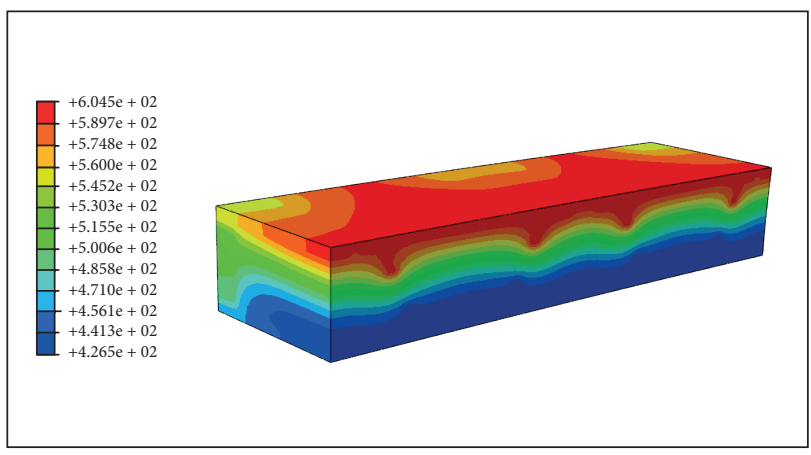

Figure 8. Temperature distribution $(K)$ at time $=50 \mathrm{~s}$. 


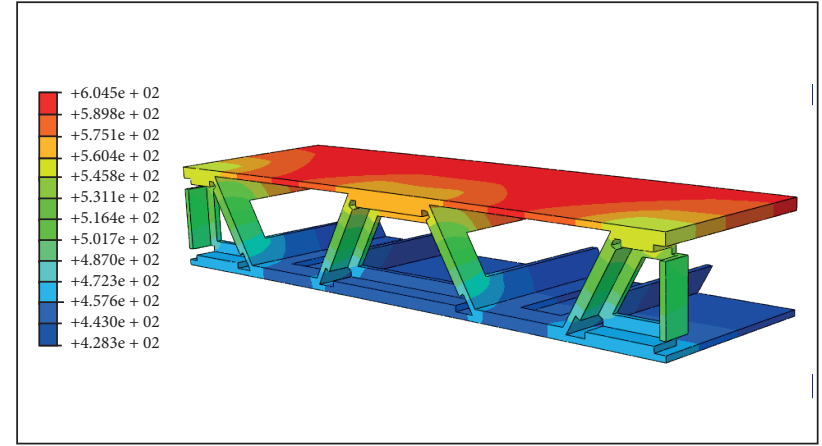

Figure 9. Temperature distribution $(K)$ at time $=50 \mathrm{~s}$ on the aluminum structure.

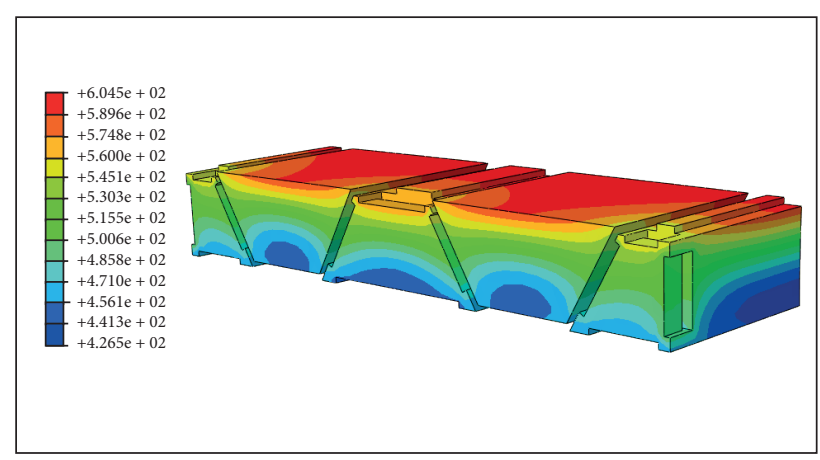

Figure 10. Temperature distribution $(K)$ at time $=50 \mathrm{~s}$ on the Saffil@ fibers.

\section{CONCLUSION}

This study shows the development of a model to study the thermo-structural behavior of a Thermally Integrated Structural Sandwich Core (TISSC) for spacecraft. Aerodynamic heating and heat conduction processes in a porous media, temperature and pressure dependent, were evaluated by previously developed and validated methodologies. A proposed design is then used for thermo-structural analyses through the finite element method. The results obtained for the proposed model and a previously designed thermo-protection system are compared. Although promising, the results have shown that the system requires further improvement, as to avoid the reinforcing structural components to behave as heat conducting elements, thus negatively affecting the insulating capability of the system. Results have also shown the high stiffness of the proposed design, in terms of its load carrying capabilities, which may be optimized and thus engender expressive mass reduction for the entire model. Therefore, although some limitations were observed in the proposed TPS, the results

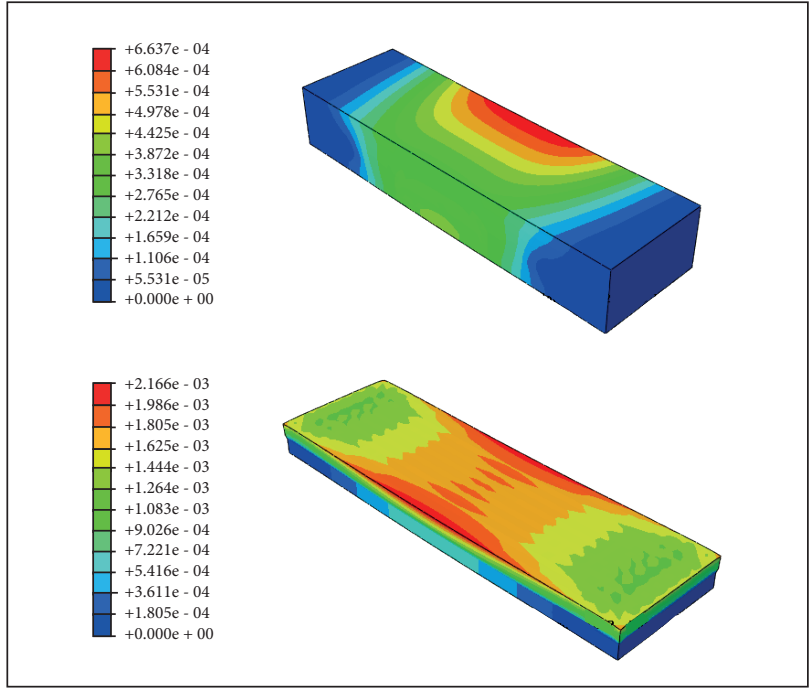

Figure 11. Displacements for each model.

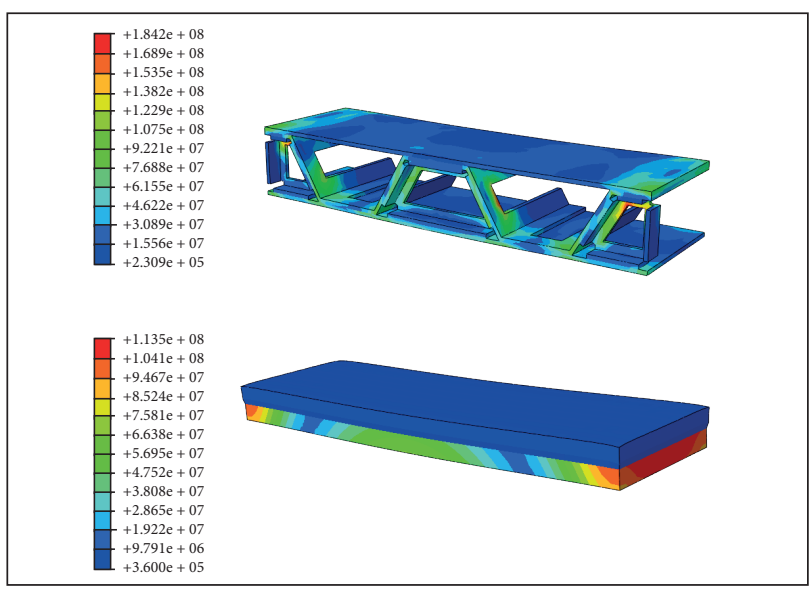

Figure 12. Stresses distributions.

presented have illustrated very well the potentiality of the employed methodology.

\section{ACKNOWLEDGEMENTS}

The financial support from the Conselho Nacional de Desenvolvimento Científico e Tecnológico (CNPq), under contract No. 479140/2011-1, is highly appreciated. The additional support received from $\mathrm{CNPq}$ under the research grant No. 309985/2013-7 is also gratefully acknowledged. The authors are also indebted to further support received from Fundação de Amparo à Pesquisa do Estado de São Paulo (FAPESP) under grant No. 2013/07375-0. 


\section{REFERENCES}

Anderson Jr., J.D., 1989, "Hyphersonic and High Temperature Gas Dynamics", McGraw-Hill, New York, USA.

Da Costa, L.E.V.L., De Mello, F.C. and Pardini, L.C., 1996, "Viability Study of Thermal Protection for SARA Platform", IAE/CTA, Technical note NT130-ASE-N/96, IAE/CTA, São José dos Campos, Brazil (in Portuguese).

Daryabeigi, K., 2000, "Design of High Temperature Multi-Layer Insulation for Reusable Vehicles", Ph.D. Thesis, University of Virginia, Charlottesville, USA.

Daryabeigi, K., 2002, "Thermal Analysis and Design Optimization of Multilayer Insulation for Reentry Aerodynamic Heating", Journal of Spacecrafts and Rockets, Vol. 39, No. 4, pp. 509-514. doi: $10.2514 / 2.3863$

Daryabeigi, K., 2003, "Heat Transfer in High Temperature Fibrous Insulation", Journal of Thermophysics and Heat Transfer, Vol. 17, No. 1, pp. 10-20. doi: 10.2514/2.6746

Daryabeigi, K., Cunnington, G.R. and Knutson, J.R., 2007 "Measurements of Heat Transfer in Unbounded Silica Fibrous Insulation and Comparison with Theory", Proceedings of the 29th International Thermal Conductivity Conference, Birmingham, USA.

Daryabeigi, K., Miller, S.D. and Cunnington, G.R., 2006, "Heat Transfer in High-Temperature Multilayer Insulation", ESA - TPS \& Hot structures. Retrieved in March 25, 2015, from http://develop. nttc.edu/sbipp/technologyportfolios/portfolios/LCRATSATPS/ Archive\%5C20080013560.pdf

Machado, H.A., 2006, "Thermal Protection for Aerodynamic Heating in the SARA Sub-orbital Platform", Proceedings of the IV National Congress of Mechanical Engineering - CONEM, Recife, Brazil (in Portuguese).

Machado, H.A., 2009, "Two-Dimensional Simulation of Multi-Layer Ablation-Conduction Problem in a Rocket TPS via an Interface Tracking Method", Proceedings of the 41st AIAA Thermophysics Conference, San Antonio, USA

Machado, H.A., 2012, "Two-Dimensional Simulation of Ablation due to Aerodynamic Heating in the SARA Sub-Obital Platform", High Temperatures. High Pressures. Retrieved in March 25, 2015, from http://www. oldcitypublishing.com/journals/hthp-home/hthp-issuecontents/hthp-volume-41-number-4-2012/hthp-41-4-p-255-271/
Machado, H.A., 2014, "Modeling Heat Transfer with Micro-Scale Natural Convection in Fibrous Insulation", Journal of the Brazilian Society of Mechanical Sciences and Engineering, Vol. 36, No. 4, pp. 847-857. doi: 10.1007/s40430-013-0107-x

Machado, H.A. and Pessoa-Filho, J.B., 2007, "Aerodynamic Heating at Hypersonic Speeds", COBEM 2007, Brasília, Brazil.

Moraes Jr., P., 1998, "Design Aspects of the Recoverable Orbital Platform SARA", Proceedings of 8th Chilean Congress of Mechanical Engineering, Concepcion, Chile.

National Oceanic and Atmospheric Administration, 1976, "U.S. Standard Atmosphere, 1976", U.S. Government Printing Office Washington, USA.

Pessoa Filho, J.B., Genaro, G., 2006, "Simulation of a Multilayer Thermal Protection System Submitted to Conditions Representative of Atmospheric Reentry", Proceedings of the 57th International Astronautical Congress, Valência, Spain

Poteet, C.C., Abu-Khajeel, H. and Hsu, S.Y., 2004, "Preliminary Thermal-Mechanical Sizing of a Metallic Thermal Protection System", Journal of Spacecraft and Rockets, Vol. 41, No. 2, pp. 173-182. doi: 10.2514/1.9174

Raed, K. and Gross, U., 2007, "Review on Gas Thermal Conductivity in Porous Materials and Knudsen Effect", Proceedings of the 29th International Thermal Conductivity Conference, Birmingham, USA.

Rakow, J.F. and Wass, A.M., 2005, "Thermal Buckling of Metal Foam Sandwich Panels for Convective Thermal Protection Systems", Journal of Spacecraft and Rockets, Vol. 42, No. 5, pp. 832-844. doi: $10.2514 / 1.9741$

Rogan, J.E. and Hurwicz, H., 1973, "High-temperature Thermal Protection Systems", In: Rohsenow, W.M., Hartnett, J.P. and Cho, Y.I. [Eds.), Handbook of Heat Transfer, McGraw-Hill, New York, USA.

Tamma, K.K. and Thornton, E.A., 1987, "Re-Entry Thermal/Structural Finite-Element Modeling/Analysis of Shuttle Configurations", Journal of Spacecraft and Rockets, Vol. 24, No. 2, pp. 101-108.

Zoby, E.V., Moss, J.N. and Sutton, K., 1981, "Approximate Convective Heat Equations Hypersonic Flows", Journal of Spacecraft and Rockets, Vol. 18, No. 1, pp. 64-70. doi: 10.2514/3.57788 